\title{
Effective interaction between star polymers
}

\author{
A. Jusufi, M. Watzlawek, and H. Löwen* \\ Institut für Theoretische Physik II, \\ Heinrich-Heine-Universität Düsseldorf, \\ Universitätsstraße 1, \\ D-40225 Düsseldorf, Germany
}

(Macromolecules 32, 4470 (1999))

\begin{abstract}
The distance-resolved effective interaction between two star polymers in a good solvent is calculated by Molecular Dynamics computer simulations. The results are compared with a pair potential proposed recently by Likos et al. [Phys. Rev. Lett. 1998, 80, 4450] which is exponentially decaying for large distances and crosses over, at the corona diameter of the star, to an ultrasoft logarithmic repulsion for small distances. Excellent agreement is found in a broad range of star arm numbers.
\end{abstract}

PACS: 61.25.Hq, 82.70.Dd, 61.20.Ja

*Also at: IFF, Forschungszentrum Jülich, D-52425 Jülich, Germany 
Star polymers are hybrids between polymer-like entities and colloidal particles establishing an important link between these different systems, for recent reviews see ref 1,2. The interpenetrability of two stars is governed by the number of arms (or functionality) $f$, i.e. the number of linear polymer chains attached to a central microscopic core. For $f=1,2$ one recovers a system composed only of linear chains while in the limit $f \rightarrow \infty$ one gets sterically-stabilized spherical colloidal particles which behave like effective hard spheres. has mainly focussed on polymer conformations of a single star. The only relevant length scale of a single star is embodied in the spatial extension of the monomers around the core as given by the so-called corona diameter $\sigma$.

In order to predict macroscopic properties of a concentrated solution of many stars, one has, however, to proceed one step further: In any statistical theory, the effective interaction between the stars is a necessary input. This interaction, in general, comprises many-body terms. For concentrations which are not too high, i.e. smaller than or comparable to the overlap concentration $\rho^{*} \equiv 1 / \sigma^{3}$, triplet and higher-order terms are small and the system is dominated by effective pairwise interactions. Recently, based on scaling theory, 9 an explicit analytical expression for the effective pair potential $V(r)$ was proposed in ref 10 . This potential combines a logarithmic form of the interaction potential for corecore separations $r$ smaller than $\sigma$ with an exponentially decaying interaction of Yukawa-form for distances $r$ larger than $\sigma$ :

$$
V(r)=\frac{5}{18} k_{B} T f^{3 / 2} \begin{cases}-\ln \left(\frac{r}{\sigma}\right)+\frac{1}{1+\sqrt{f} / 2} & ; r \leq \sigma \\ \frac{1}{1+\sqrt{f} / 2}\left(\frac{\sigma}{r}\right) \exp \left(-\frac{\sqrt{f}}{2 \sigma}(r-\sigma)\right) & ; r>\sigma\end{cases}
$$

Note that the potential strength simply scales with the thermal energy $k_{B} T$ since the repulsion between the stars is of purely entropic origin having a good solvent in mind. Both, the potential in eq 1 and its associated force $F(r)=-d V(r) / d r$ are continuous at $r=\sigma$, but $F(r)$ has an artificial cusp at $r=\sigma$. The prefactor of the logarithm is fixed by scaling theory $\mathrm{g}$ while the exponential decay length $2 \sigma / \sqrt{f}$ is the diameter of the outermost blob within the Daoud-Cotton model for one star polymer.

For an arm number of $f=18$, this potential was tested against neutron scattering data and reasonable agreement was found.10 Further experimental support comes from shear moduli measurements in the crystalline phase of manyarm-micelles.11 Still, the scaling theory assumptions are strictly speaking only justified for core-core distances $r$ much smaller than $\sigma$, and the exponential decay length of the outermost blob size is an heuristic assumption. Hence the validity of the potential for arbitrary arm numbers can be questioned. In this paper, we test the pair potential against a microscopic model, resolving the monomers of the chains, by extensive Molecular Dynamics computer simulations. To be specific, we use a simulation model for star polymers developed by Grest et al., 6 which was 
applied in previous studies for single stars, and generalize it to a situation with two stars, which is the minimal set-up to extract information about the effective interaction between two stars. The distance-resolved interaction force $F(r)$ is calculated for arm numbers $f$ ranging from $f=5$ to $f=50$. Each arm contains $N$ monomers where $N$ is varied from 50 to 200. As a result, we confirm the phenomenological interaction potential in eq 1; our simulation results are in perfect quantitative agreement with the theoretical prediction. This important result enables a mapping of a star polymer solution onto a classical one-component fluider interacting via the effective ultra-soft pair potential of eq 1, provided the star concentration does not exceed $\rho^{*}$. This picture was anticipated in recent work, calculating the anomalous structure factor of star polvmer solutions 13 and the unusual phase diagram including re-entrant melting 14 and anisotropic crystal structures 14 So, our present work provides a theoretical justification of all these previous studies.

Let us first describe the simulation model:6 Each polymer arm consists of $N$ effective monomers or "beads" interacting via a purely repulsive Lennard-Joneslike potential $V_{0}(r)$, where $r$ is the separation of the beads. $V_{0}(r)$ is obtained from the usual Lennard-Jones potential $V_{L J}(r)$ by cutting $V_{L J}(r)$ at the position of the potential minimum $r_{m}=2^{1 / 6} \sigma_{L J}$ and by shifting it by the constant value $V_{L J}\left(r_{m}\right)$ in order to obtain $V_{0}\left(r_{m}\right)=0$ :

$$
V_{0}(r)= \begin{cases}4 \epsilon\left[\left(\frac{\sigma_{L, J}}{r}\right)^{12}-\left(\frac{\sigma_{L, J}}{r}\right)^{6}+\frac{1}{4}\right] & ; r \leq 2^{1 / 6} \sigma_{L J} \\ 0 & ; r>2^{1 / 6} \sigma_{L J}\end{cases}
$$

Here, $\epsilon$ sets the energy scale and $\sigma_{L J}$ the length scale of the beads. The pure repulsion implies that we are dealing with a good solvent. For neighbouring beads along the chains, the attractive FENE-potential6 $V_{c h}(r)$ is added to the interaction

$$
V_{c h}= \begin{cases}-15 \epsilon\left(\frac{R_{0}}{\sigma_{L J}}\right)^{2} \ln \left[1-\left(\frac{r}{R_{0}}\right)^{2}\right] & ; r \leq R_{0} \\ \infty & ; r>R_{0}\end{cases}
$$

This interaction diverges at $r=R_{0}$, which determines the maximum relative displacement of two neighbouring beads. Henceforth, we fix $R_{0}$ to be $1.5 \sigma_{L J}$. Then the total potential $V_{0}(r)+V_{c h}(r)$ between neighbouring monomers has a minimum at $r \approx 0.97 \sigma_{L J}$. Furthermore, the central core particles of the two stars have a finite hard core radius $R_{c}$, and all monomers are interacting with the core particles via a modified repulsive interaction potential $V_{0}^{c}(r)$. The introduction of a small hard core of the central particles of the stars is necessary to accommodate the large number of arms at small distances from the core. explicitly for the potential

$$
V_{0}^{c}(r)= \begin{cases}\infty & ; r \leq R_{c} \\ V_{0}\left(r-R_{c}\right) & ; r>R_{c}\end{cases}
$$


In addition, the innermost monomers of each arm are interacting with their core via an attractive potential which is given by

$$
V_{c h}^{c}(r)= \begin{cases}\infty & ; r \leq R_{c} \\ V_{c h}\left(r-R_{c}\right) & ; r>R_{c}\end{cases}
$$

We note that exactly this simulation model was already used by Grest et al. in their simulations of single star polymers. In our simulations, the centers of the two stars are fixed at positions $\vec{R}_{1}$ and $\vec{R}_{2}$ with a given distance $r=\left|\vec{R}_{1}-\vec{R}_{2}\right|$. The total number of mobile monomers is $2 f N$, which limits our studies to small $f$ and small $N$. In all simulations, the system is held at fixed temperature $T=1.2 \epsilon / k_{B}$. Under these circumstances, the effective force $\vec{F}_{i}$ acting on the $i$ th star center is given as a canonical average

$$
\vec{F}_{i}=\left\langle-\vec{\nabla}_{\vec{R}_{i}}\left(\sum_{k=1}^{2 f N} V_{0}^{c}\left(\left|\vec{r}_{k}-\vec{R}_{i}\right|\right)+\sum_{l=1}^{f} V_{c h}^{c}\left(\left|\vec{r}_{l}-\vec{R}_{i}\right|\right)\right)\right\rangle,
$$

where in the first sum the repulsive interactions of the core with all $2 f N$ monomers in the system are considered, whereas the second sum only accounts for the attractive interactions with the $f$ innermost monomers of the chains attached to the $i$ th center. Obviously, due to symmetry, $\vec{F}_{1}=-\vec{F}_{2}$. We use standard Molecular Dynamics simulation $\$ 15$ to equilibrate the monomers and perform the statistical average $\langle\ldots\rangle$ over the monomers for the forces on the star centers. The timestep is typically $\delta t=0.002 \tau$ (with $\tau=\sqrt{m \sigma_{L J}^{2} / \epsilon}$ being the associated time unit and $m$ the monomer mass) and typically 120000 steps are used for equilibration and up to $t_{\max } / \delta t=500000$ steps were simulated to gather statistics. It was carefully checked by monitoring the internal energy that the system had equilibrated. A typical snapshot of two stars after equilibration is shown in Figure 1. As can be seen, the monomers of one star do not penetrate much into the central region of the other star.

In order to check the code, we performed simulations of single stars changing the arm numbers between $f=5$ and $f=50$ and the monomer numbers from $N=50$ to $N=200$. The corresponding results for the radius of gyration $R_{G}^{2}=\frac{1}{f N} \sum_{i=1}^{f N}\left(\vec{r}_{i}-\vec{r}_{C M}\right)^{2}$ (where $\vec{r}_{C M}$ is the center of mass of the whole star) and the density profile of the monomers are in very good agreement with the results given in ref 6 and are well described by the scaling theory of Daoud and Cotton.5 For a detailed list of the simulation parameters and the results for $R_{G}$, obtained from these single star simulations, see Table $\mathbb{\text { I. }}$.

It should be noted that the effective forces on the star centers are the gradient of the effective star-star potential. This effective potential, however, differs in general from the monomer averaged potential energy of the star centers. 16 We therefore had to calculate the averaged forces $\vec{F}_{i}(i=1,2)$ from our two star simulations to compare with the theoretical force as calculated from eq 11. In doing this, two difficulties are arising: i) The corona diameter $\sigma$, which is the 
relevant length scale in the potential of eq 1 , is not known a priori. ii) In contrast to the theory, there is a finite core size $R_{c}$ in our simulation model.

As regards the first difficulty, $\sigma$ is usually defined as the typical maximum range where a scaling behaviour of the monomer density around a single star center holds. 9 A statistical definition of $\sigma$, however, is missing. On the other hand, the radius of gyration $R_{G}$ has a clear definition as a canonical average, which can be calculated directly in simulations. We therefore use $R_{G}$, which was calculated in the single star simulations, as basic length scale for our simulation data and fit these data for $F=\left|\vec{F}_{i}\right|(i=1,2)$ to the theoretical prediction for $F(r)$ using the least-square method and treating $\sigma$ as the single fit parameter. Afterwards we check how the optimal value for $\sigma$ scales with $R_{G}$ as obtained from the single star simulations. The procedure is consistent if the ratio $\lambda=\sigma / 2 R_{G}$ is independent of $f$. The second difficulty is resolved as follows: A logarithmic potential for $r<\sigma$ implies that the data should fall on a straight line crossing the origin if one plots the inverse force, $1 / F$, versus $r$ inside the corona diameter. A typical plot is given in Figure 2. In fact, the data fall on a straight line. Extrapolating the data, however, one does not hit the origin. The divergence of the force occurs already at a finite distance $2 R_{d} \approx 2 R_{c}+\sigma_{L J}$ which clearly has to be attributed to the presence of the finite core in the simulations. We note that both, $R_{c}$ and $R_{d}$, are microscopic length scales and of same order of magnitude (see Table (1), thus being not relevant for the macroscopic length $\sigma$ in the scaling regime. We therefore normalize our distances by subtracting $2 R_{d}$, thus matching the divergence of the force properly. We emphasize that the slope of the straight line is in very good agreement with the theoretical prediction, see again Figure 2 . This implies that the theoretical prefactor $\frac{5}{18} f^{3 / 2} k_{B} T$ in eq 1 is confirmed by the computer simulations. In Figure 3, we show the effective force versus distance for five different arm numbers $f$ and two monomer numbers $N$. The agreement with the theory is convincing for all $f$ and $N$. The consistency of our fitting procedure

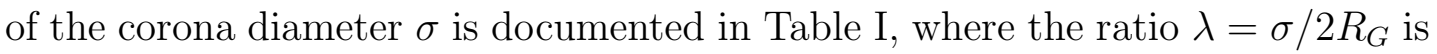
given for different $f$ and $N$. We find $\lambda \approx 0.65$ independent of $f$. This value also coincides with the value used in ref 10 to fit experimental data for $f=18$. We further note that $\lambda$ is independent of $N$, consistent with scaling theory. Finally, we prove the exponential decay of the force for distances larger than $\sigma$ by plotting the logarithm of the force versus distance in Figure 4 for one typical example. One clearly sees the crossover of the inner-core data to a straight line outside the core. The slope is consistent with the theoretical one as determined by the outermost blob size.

In conclusion, we have verified the ultra-soft pair interaction for star polymers by direct molecular simulations. It is straightforward to generalize the method to two stars confined in a periodically-repeated cubic cell in order to estimate the shrinking of the corona diameter due to a finite star density. Also, similar to charged colloids, 17 triplets of stars should be considered to investigate the 
importance of triplet interactions. Our future work lies along these directions.

We thank M. Schmidt and C. N. Likos for helpful remarks.

M. W. thanks the Deutsche Forschungsgemeinschaft for support within SFB 237. 


\section{References}

(1) Grest, G. S.; Fetters, L. J.; Huang, J. S.; Richter, D. Adv. Chem. Phys. 1996, $X C I V, 67$.

(2) Gast, A. P. Langmuir 1996, 12, 4060.

(3) Pusey, P. N. In Liquids, Freezing and Glass Transition, Hansen, J. P., Levesque, D. L., Zinn-Justin, J., Eds.; North Holland: Amsterdam, 1991.

(4) Löwen, H. Phys. Rep. 1994, 237, 249.

(5) Daoud, M.; Cotton, J. P. J. Physique 1982, 43, 531.

(6) Grest, G. S.; Kremer, K.; Witten, T. A. Macromolecules 1987, 20, 1376. Grest, G. S. Macromolecules 1994, 27, 3493.

(7) Shida, K.; Ohno, K.; Kimura, M.; Kawazoe, Y.; Nakamura, Y. Macromolecules 1998, 31, 2343. Sikorski, A.; Romiszowski, P. J. Chem. Phys. 1998, 109, 6169. Batoulis, J.; Kremer, K. Macromolecules 1989, 22, 4277. Freire, J. J.; Pla, J.; Rey, A.; Prats, R. Macromolecules 1986, 19, 452. Ishizu, K.; Ono, T.; Uchida, S. Macromol. Chem. Phys 1997, 198, 3255. Forni, A.; Ganazzoli, F.; Vacatello, M. Macromolecules 1997, 30, 4737.

(8) Willner, L.; Jucknischke, O.; Richter, D.; Roovers, J.; Zhou, L.-L.; Toporowski, P. M.; Fetters, L. J.; Huang, J. S.; Lin, M. Y.; Hadjichristidis, N. Macromolecules 1994, 27, 3821. Richter, D.; Jucknischke, O.; Willner, L.; Fetters, L. J.; Lin, M.; Huang, J. S.; Roovers, J.; Toporovski, C.; Zhou, L. L. J. Physique IV 1993, 3, 3. Willner, L.; Jucknischke, O.; Richter, D. ; Farago, B.; Fetters, L. J.; Huang, J. S. Europhys. Lett. 1992, 19, 297.

(9) Witten, T. A.; Pincus, P. A. Macromolecules 1986, 19, 2509. Witten, T. A.; Pincus, P. A.; Cates, M. E. Europhys. Lett. 1986, 2, 137.

(10) Likos, C. N.; Löwen, H.; Watzlawek, M.; Abbas, B.; Jucknischke, O.; Allgaier, J.; Richter, D. Phys. Rev. Lett. 1998, 80, 4450.

(11) Buitenhuis, J.; Förster, S. J. Chem. Phys. 1997 107, 262.

(12) See, e.g.: Hansen, J. P.; McDonald, I. R. Theory of Simple Liquids, 2nd ed.; Academic Press: London, 1986.

(13) Watzlawek, M.; Löwen, H.; Likos, C. N. J. Phys. Condensed Matter 1998, $10,8189$.

(14) Watzlawek, M.; Likos, C. N.; Löwen, H. Phys. Rev. Lett 1999, 82, 5289. 
(15) See, e.g.: Allen M. P.; Tildesley, D. J. Computer Simulation of Liquids; Clarendon Press: Oxford, 1989.

(16) Löwen, H. Progr. Colloid Polym. Sci. 1998, 110, 12.

(17) Löwen, H.; Allahyarov, E. J. Phys. Condensed Matter 1998, 10, 4147. 


\section{Tables}

\begin{tabular}{|c|c|c|c|c|c|c|c|}
\hline$f$ & $N$ & $\delta t / \tau$ & $t_{\max } / \delta t$ & $R_{G} / \sigma_{L J}$ & $\left(R_{c} / \sigma_{L J}+1 / 2\right.$ & $R_{d} / \sigma_{L J}$ & $\lambda$ \\
\hline 5 & 100 & 0.004 & 500000 & 13.53 & 0.65 & 1.39 & 0.61 \\
10 & 50 & 0.003 & 400000 & 10.37 & 1.1 & 1.21 & 0.66 \\
10 & 100 & 0.003 & 400000 & 16.18 & 1.1 & 0.89 & 0.64 \\
10 & 150 & 0.003 & 400000 & 19.71 & 1.1 & 1.31 & 0.60 \\
10 & 200 & 0.003 & 400000 & 24.52 & 1.1 & 1.42 & 0.67 \\
18 & 50 & 0.002 & 350000 & 11.19 & 1.25 & 1.38 & 0.68 \\
18 & 100 & 0.002 & 350000 & 17.10 & 1.25 & 1.64 & 0.65 \\
30 & 50 & 0.002 & 350000 & 12.22 & 1.6 & 1.89 & 0.66 \\
50 & 50 & 0.002 & 350000 & 13.35 & 1.8 & 2.40 & 0.69 \\
\hline
\end{tabular}

Table I: List of the simulation parameters and the corresponding results for $R_{G}$ and $\lambda=\sigma / 2 R_{G}$.

\section{Figure captions}

Figure 1: Typical configuration for two stars with $f=10$ and $N=50$. The distance between the central core particles, which are shown as big black spheres, is $r=5.2 \sigma_{L J}$. The gray and light gray monomers are belonging to the first and second star respectively.

Figure 2: $\quad$ Reduced inverse force $k_{B} T /\left(F R_{G}\right)$ between the centers of two star polymers (for $f=10$ and $N=50$ ) versus reduced distance $r / R_{G}$. The error bars were obtained by averaging over the results of 8 independent simulations.

Figure 3: Simulation results (symbols) and theoretical results (lines) for the reduced effective force $F R_{G} / k_{B} T$ versus reduced distance $\left(r-2 R_{d}\right) / R_{G}$. a) for $f=5,10,18$ and $N=100, \mathrm{~b})$ for $f=18,30,50$ and $N=50$.

Figure 4: Logarithm of the reduced force $\ln \left(F R_{G} / k_{B} T\right)$ versus reduced distance $\left(r-2 R_{d}\right) / R_{G}$ for $f=10$ and $N=50$. The error bars were obtained by averaging over the results of 8 independent simulations. 


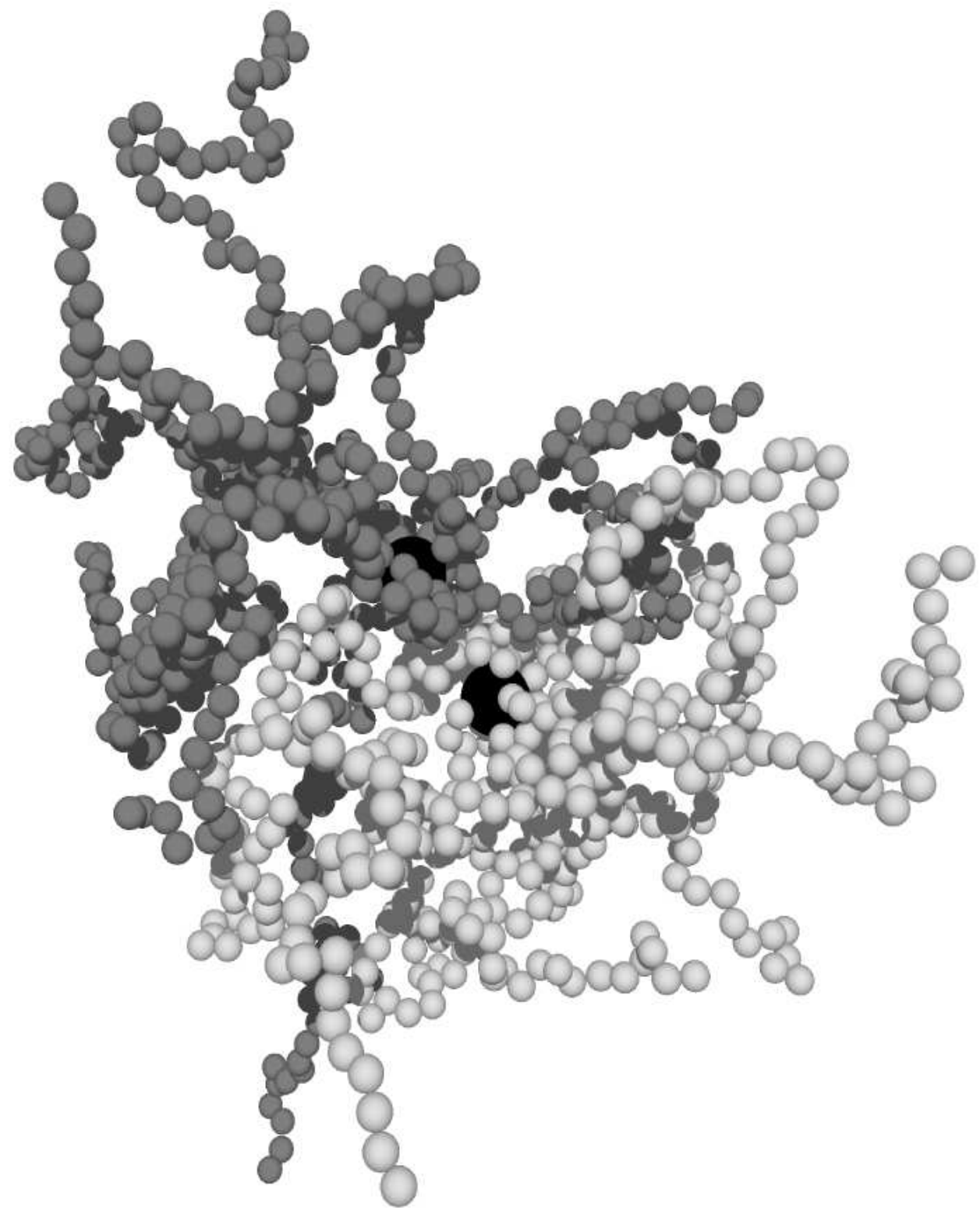




\section{Jusufi et al., fig.2}

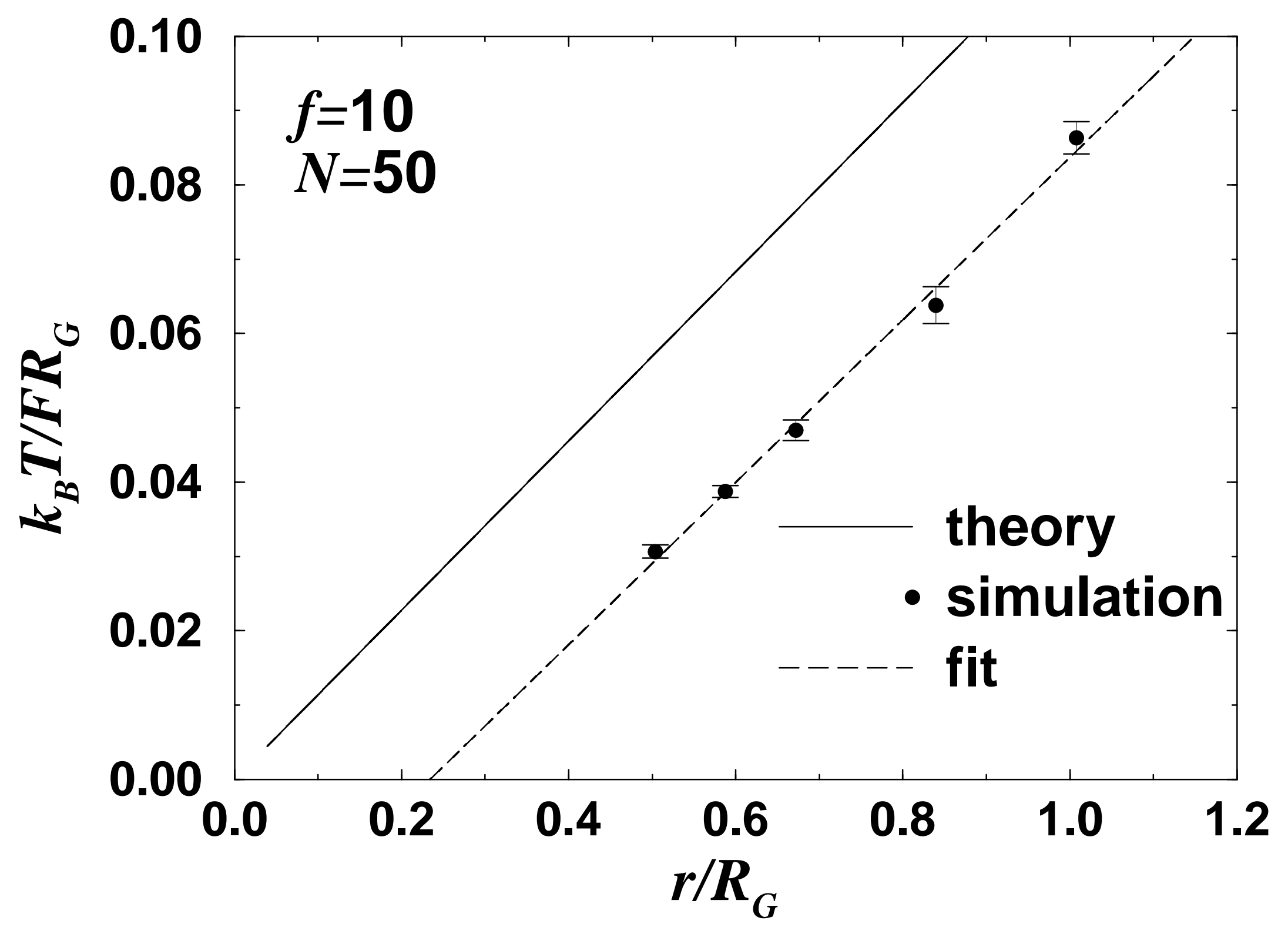


Jusufi et al., fig.3a

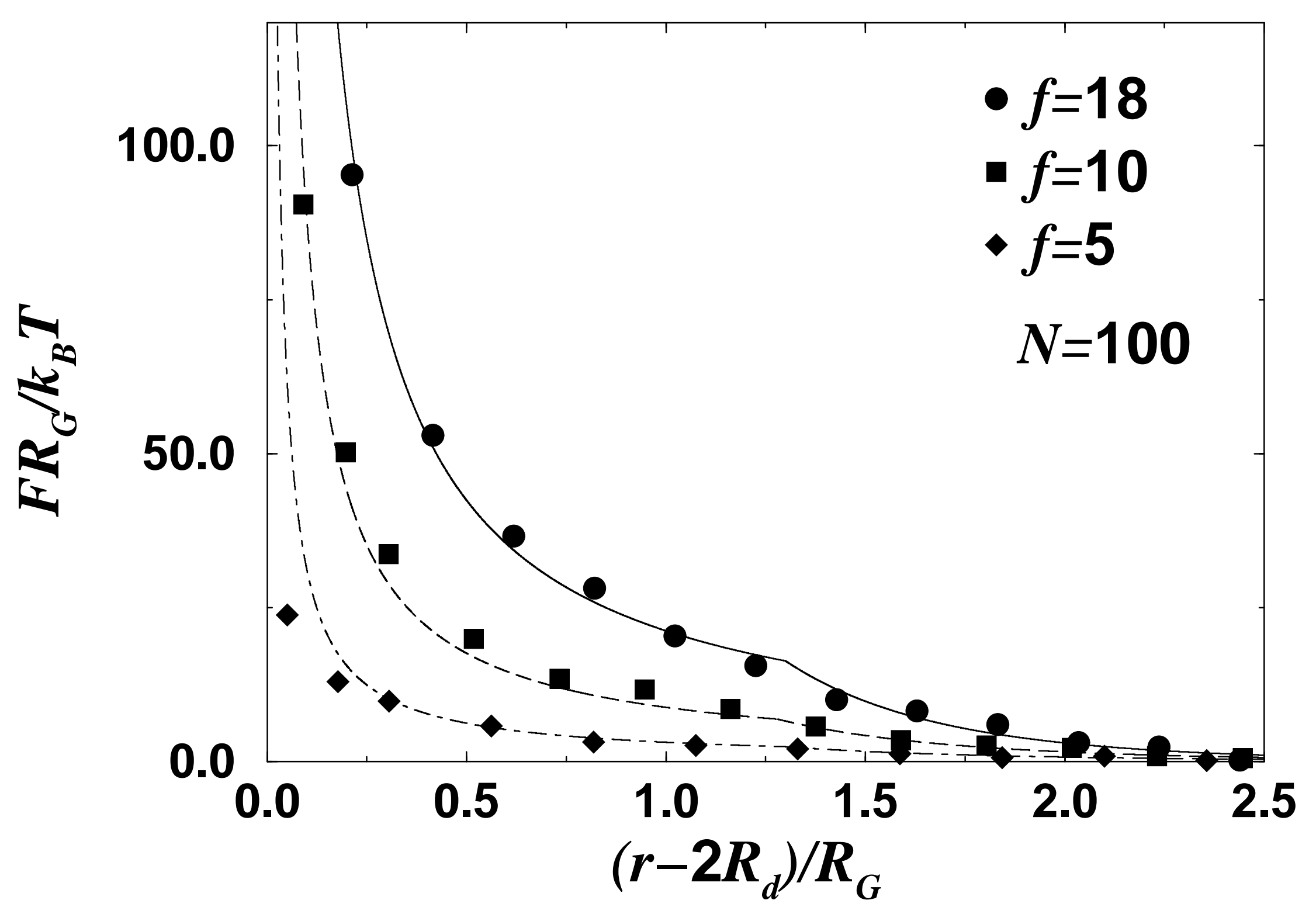




\section{Jusufi et al., fig.3b}

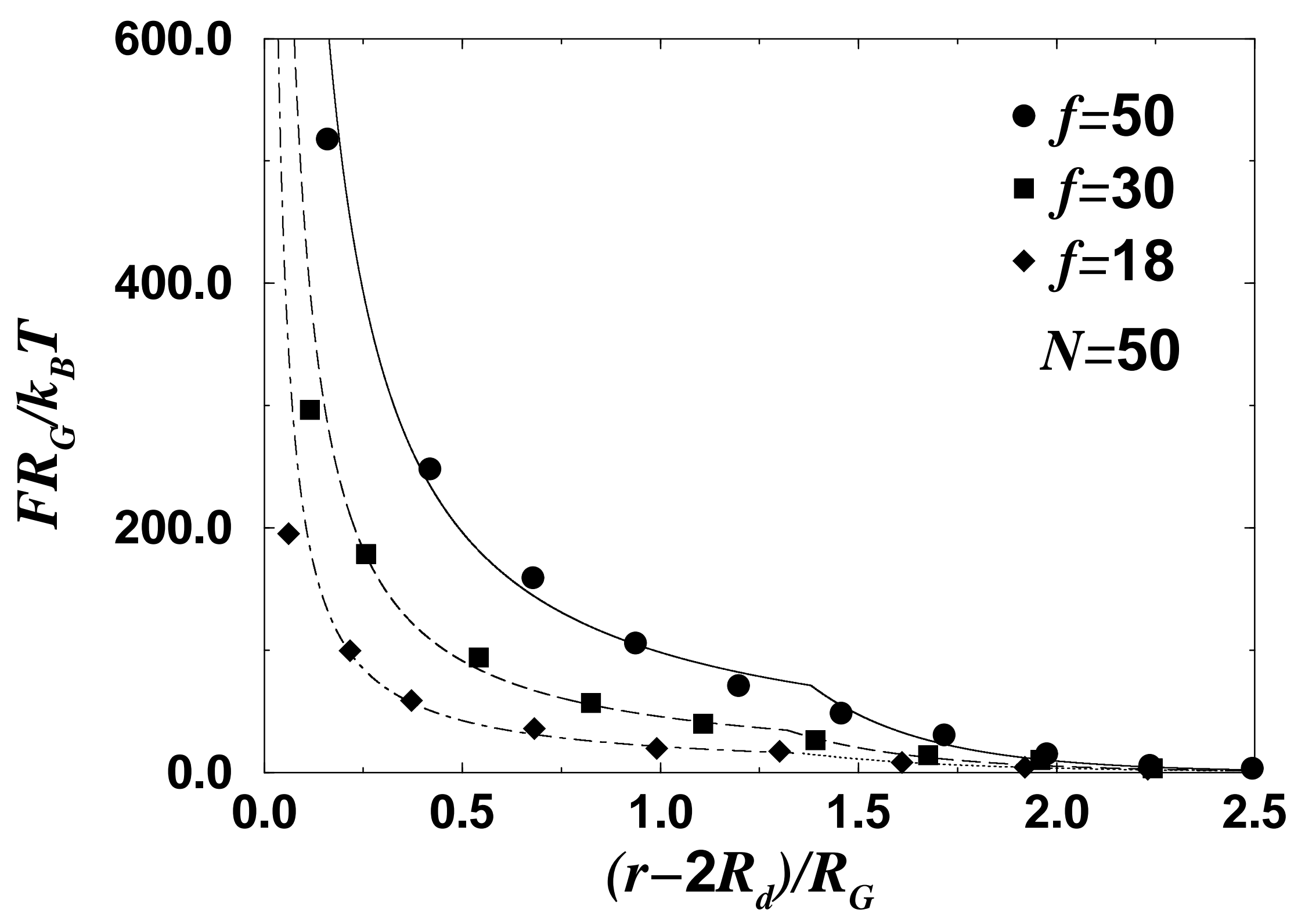




\section{Jusufi et al., fig.4}

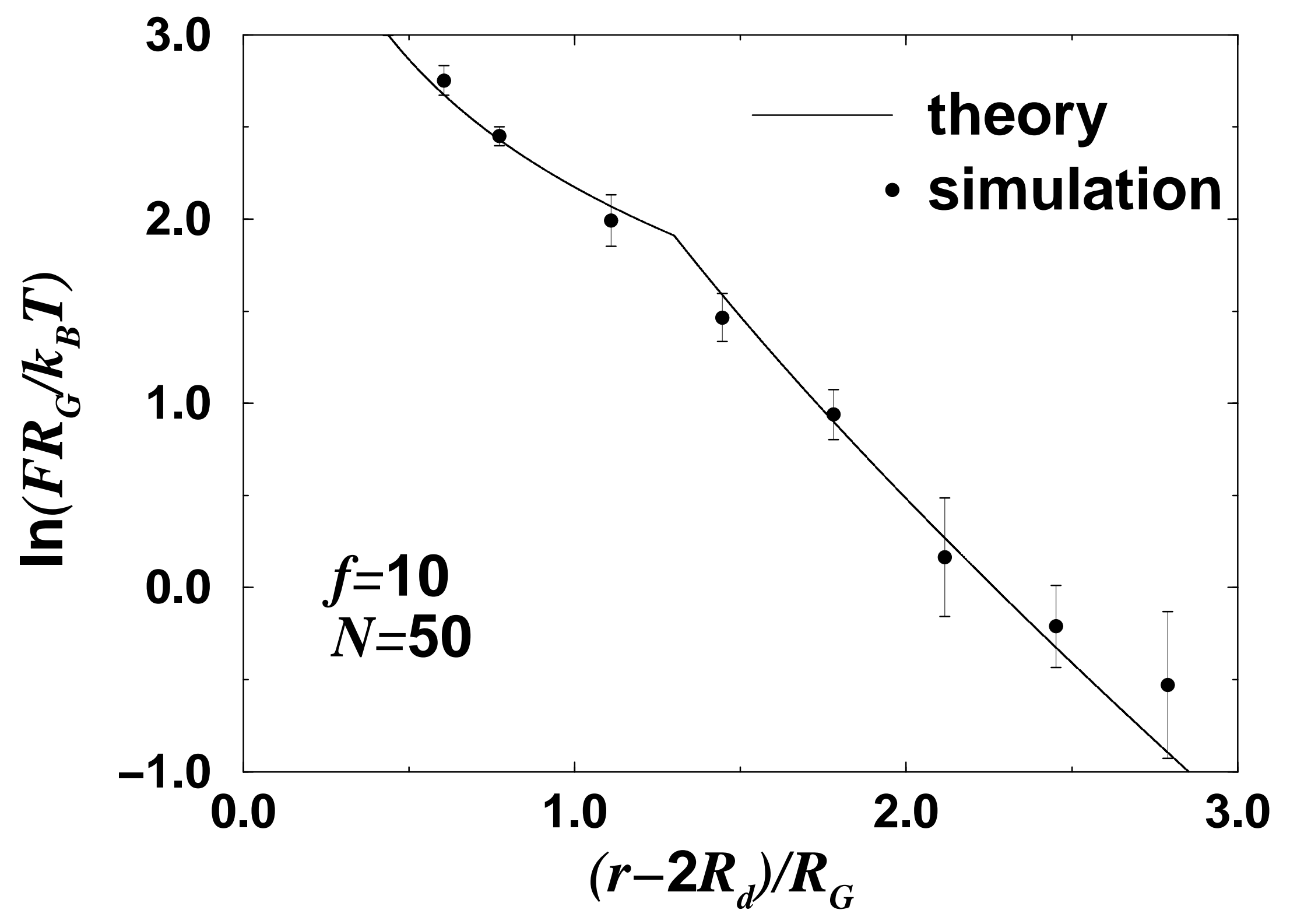

